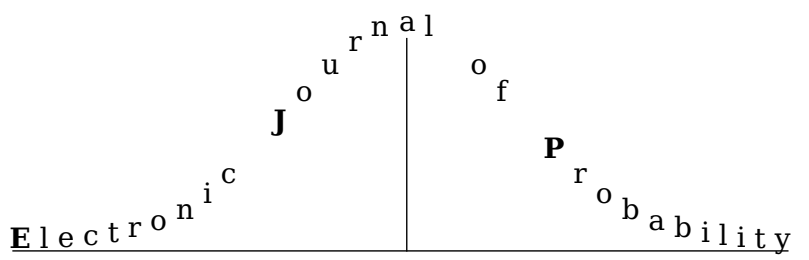

Electron. J. Probab. 25 (2020), article no. 117, 1-24.

ISSN: 1083-6489 https://doi.org/10.1214/20-EJP509

\title{
Limit theorems for integrated trawl processes with symmetric Lévy bases
}

\author{
Anna Talarczyk* $\quad$ Eukasz Treszczotko ${ }^{\dagger}$
}

\begin{abstract}
We study long time behavior of integrated trawl processes introduced by BarndorffNielsen (2011). The trawl processes form a class of stationary infinitely divisible processes, they are described by an infinitely divisible random measure (Lévy base) and a family of shifts of a fixed set (trawl). We assume that the Lévy base is symmetric and homogeneous and that the trawl set is determined by the trawl function that decays slowly. Depending on the geometry of the trawl set and on the Lévy measure corresponding to the Lévy base we obtain various types of limits in law of the normalized integrated trawl processes for large times. The limit processes are always stable and self-similar with stationary increments. In some cases they have independent increments - they are stable Lévy processes where the index of stability depends on the parameters of the model. We show that stable limits with stability index smaller than 2 may appear even in cases when the underlying Lévy base has all its moments finite. In other cases, the limit process has dependent increments and it may be considered as a new extension of fractional Brownian motion to the class of stable processes.
\end{abstract}

Keywords: trawl processes; Lévy bases; stable processes; self-similar processes; Lévy processes; limit theorems; fractional Brownian motion; infinite divisibility.

MSC2020 subject classifications: Primary 60G51; 60F17, Secondary 60F05; 60G52; 60G18; $60 \mathrm{G} 57$.

Submitted to EJP on October 17, 2019, final version accepted on August 10, 2020.

\section{Introduction}

Trawl processes form a class of stationary infinitely divisible processes. They first appeared in the work of Wolpert and Taqqu [15] where the term "upstairs representation"

\footnotetext{
*Institute of Mathematics, University of Warsaw, ul. Banacha 2, 02-097 Warsaw, Poland.

E-mail: annatal@mimuw. edu.pl. Research supported in part by National Science Center, Poland, grant 2016/23/B/ST1/00492.

${ }^{\dagger}$ Institute of Mathematics, University of Warsaw, ul. Banacha 2, 02-097 Warsaw, Poland.

E-mail: l.treszczotko@mimuw. edu.pl. Research supported in part by National Science Center, Poland, grant 2017/25/N/ST1/00368.
} 
was used. Then, they were independently introduced by Barndoff-Nielsen in [1] under the name of trawl processes. These processes were studied further in [5], [3], [10] and [14]. Trawl processes form a subclass of so-called ambit processes, which are useful in modelling of various phenomena, for example turbulence in physics, tumor growth in medicine, some aspects of financial mathematics, in particular related to volatility/intermittency. The book [4] contains an exposition on trawl processes, general ambit processes and their applications. Discrete time counterparts of trawl processes were investigated in [9].

Trawl processes are defined in the following way: suppose that $\Lambda$ is a homogenous Lévy basis on $\mathbb{R}^{2}$, that is, an infinitely divisible independently scattered random measure on $\mathbb{R}^{2}$, and let $A$ be a Borel subset of $\mathbb{R}^{2}$ with finite Lebesgue measure. Let $A_{t}$ denote $A$ shifted by the vector $(0, t), A_{t}:=A+(t, 0)$. A trawl process is the process of the form

$$
X_{t}=\Lambda\left(A_{t}\right), \quad t \in \mathbb{R} .
$$

The set $A$ is called a trawl. Since $\Lambda$ is homogeneous and infinitely divisible, the process $\left(X_{t}\right)_{t \geq 0}$ is stationary and infinitely divisible. To any Lévy basis there corresponds a Lévy process $L=(L(t))_{t \geq 0}$ (a process with stationary and independent increments). $L$ can be taken e.g. as $L(t):=\Lambda([0, t] \times[0,1])$. The process $L$ is called Lévy seed. The one-dimensional distributions of $X_{t}$ are determined by the choice of the Lévy seed and the dependence structure of a trawl process depends on the shape of the set $A$.

The processes of the form (1.1) are interesting mainly because they form a large class of processes that allows to model independently of each other the marginal distributions and the dependence structure.

Typically, the set $A$ is determined by a trawl function $g:[0, \infty) \rightarrow[0, \infty)$ with $\int_{0}^{\infty} g(s) d s<\infty$. More precisely we define

$$
A:=\{(x, y): x \leq 0, y \leq g(-x)\}
$$

and then

$$
A_{t}=A+(t, 0)=\{(x, y): x \leq t, 0 \leq y \leq g(t-x)\} .
$$

In the present paper we will investigate the behaviour of the integrated trawl process. More precisely, we study the convergence in law of the rescaled integrated trawl process

$$
Y_{T}(t)=\frac{1}{F_{T}} \int_{0}^{T t} X_{s} d s
$$

as $T \rightarrow \infty$, where $F_{T}$ is an appropriate norming, chosen so that there exists a non-trivial limit in law.

It seems quite clear that if $g$ vanishes sufficiently quickly, then the increments of $Y_{T}$ become asymptotically independent. A more interesting situation is when $g$ decays slowly, which will be the object of the current study. We will assume that the function $g$ in (1.2) is strictly decreasing, integrable and has a continuous derivative, that for large $t$ behaves as const $\times t^{-2-\gamma}$, for some $0<\gamma<1$. Typically one can think of $g$ of the form $C(1+t)^{-1-\gamma}$. It is known (see [10]) that if $g$ is regularly varying at infinity with index $-1-\gamma$, with $\gamma \in(0,1)$, then the corresponding trawl process is long range dependent.

Depending on the interplay between the type of decay of $g$ and the underlying Lévy measure of the Lévy base $\Lambda$ we show that the limit in law of (1.3) can be either a continuous stable process with dependent increments or a stable Lévy process with index of stability depending on the parameters of the model. 


\subsection{Background}

Let us briefly describe the history of this problem and related results. In [10] Grahovac, Leonenko and Taqqu studied the behaviour of the integrated trawl process

$$
Z(t)=\int_{0}^{t} X_{s} d s
$$

with assumption that the trawl function was regularly varying at infinity with the exponent $1+\gamma$ where $0<\gamma<1$. It was also assumed that the underlying Lévy seed process had exponential moments.

It was shown that if one defines

$$
\tau_{*}(q):=\lim _{t \rightarrow \infty} \frac{\log \left(\mathbb{E}|Z(t)|^{q}\right)}{\log t},
$$

then there exists $q^{*} \geq 0$ such that for any $q^{*} \leq q$ one has $\tau_{*}(q)=q-\gamma$. This implies that for $q^{*} \leq p<q$

$$
\frac{\tau_{*}(p)}{p}<\frac{\tau_{*}(q)}{q} .
$$

This property is known as intermittency. In particular, intermittency implies that if the process $Y_{T}$ given by (1.3) converges in the sense of finite dimensional distributions as $T \rightarrow \infty$ to some process $\left(Z_{t}\right)_{t \geq 0}$, then it is impossible to have convergence of all moments

$$
\lim _{T \rightarrow \infty} \mathbb{E}\left|\frac{Y_{T}(t)}{F_{T}}\right|^{q}=\mathbb{E}|Z(t)|^{q}
$$

for all $q>q^{*}$ and $t>0$. This follows form the fact that $Z$ would have to be self-similar with index $H$, i.e., $(Z(c t))_{t \geq 0} \stackrel{d}{=} c^{H}(Z(t))_{\geq 0}$ for all $c>0$, and $F_{T}$ of the form $F_{T}=T^{H} L(T)$ for some $H>0$ and a function $L$ which is slowly varying at $+\infty$, hence $\frac{\tau(q)}{q}$ would have to be constant. A natural question for us was to try to identify the limit process. Indeed, as we shall see later, this corresponds to the situation of our Theorem 2.7, where the limit process of (1.3) is a stable process, with the stability parameter depending on the type of decay of the trawl function, even though $X_{t}$ has all moments finite.

Another related paper is [9], where discrete time, integer valued trawl processes have been considered. They are of the form

$$
X_{k}=\sum_{j=0}^{\infty} \gamma_{k-j}\left(a_{j}\right) \quad k \in \mathbb{Z}
$$

where $\gamma_{k}=\left(\gamma_{k}(u)\right)_{u \in \mathbb{R}}$ are i.i.d. copies of some process $\gamma=(\gamma(u))_{u \in \mathbb{R}}$ with $\gamma(u) \rightarrow 0$ in probability as $u \rightarrow 0$, and $a_{j} \in \mathbb{R}, j \in \mathbb{N}, \lim _{j \rightarrow \infty} a_{j}=0$. $\left(X_{k}\right)$ is the trawl process corresponding to the seed process $\gamma$. In [9] the behaviour of the process of partial sums

$$
S_{n}(t)=\frac{1}{F_{n}} \sum_{k=1}^{\lceil n t\rceil}(X(k)-\mathbb{E} X(k))
$$

was investigated as $n \rightarrow \infty$ with an appropriate norming $F_{n}$. The authors considered the seed process with finite variance. Depending on the behaviour of the seed process and the trawl function $\left(a_{j}\right)$ various limits are obtained, either Gaussian limits: fractional Brownian motion and Brownian motion, or stable limits: $\alpha$-stable Lévy process. In particular, long memory trawl function $a_{j} \sim j^{-\alpha}, \alpha \in(1,2)$ and the standard Poisson seed process $\gamma$ leads to $\alpha$-stable Lévy process, even though with different norming the covariances converge to those of a fractional Brownian motion. 


\subsection{Description of the results}

In this section we briefly describe our results. For precise statements of our theorems in their general form see Section 2. We study the behaviour of the rescaled integrated trawl process $Y_{T}$ given by (1.3). Our basic assumption is that $A_{t}$ is of the form (1.2) with the trawl function $g:[0, \infty) \rightarrow[0, \infty)$ which is integrable, strictly decreasing, has a continuous first derivative such that for large $t$ we have $g^{\prime}(t) \sim-$ const $\times t^{-2-\gamma}$ for some $0<\gamma<1$ (this corresponds to the assumption in [9] that $a_{j} \sim j^{-1-\gamma}$ and to the assumptions made in [10]). In the latter paper the assumptions on $g$ were slightly less restrictive $-g^{\prime}$ regularly varying at $+\infty$, but no limit in law theorems were established.

We consider a homogeneous Lévy base $\Lambda$ such that for every $A \in \mathcal{B}\left(\mathbb{R}^{2}\right)$ (a Borel subset of $\mathbb{R}^{2}$ ) with finite Lebesgue measure, $\Lambda(A)$ is symmetric and does not have a Gaussian component, that is

$$
\mathbb{E} \exp (i \theta \Lambda(A))=\exp \{-|A| \psi(\theta)\},
$$

where $|A|$ is the Lebesgue measure of $A, \psi$ is the Lévy exponent

$$
\psi(\theta)=\int_{\mathbb{R}}\left(1-e^{i \theta y}+i \theta u \mathbb{1}_{\{|y|<1\}}\right) \nu(d y),
$$

and $\nu$ is a Lévy measure, i.e., a Borel measure on $\mathbb{R}$ satisfying

$$
\int_{\mathbb{R}} 1 \wedge|y|^{2} \nu(d y)<\infty
$$

with $\nu(\{0\})=0$. We assume that $\nu$ is symmetric, hence (1.7) can be written as

$$
\psi(\theta)=\int_{\mathbb{R}}(1-\cos (\theta y)) \nu(d y), \quad \theta \in \mathbb{R} .
$$

The assumption of symmetry simplifies some parts of the proofs, as well as assumptions of the theorems formulated below, but we expect that it is not essential and it should be possible to obtain analogous results in the non symmetric case, with the limit processes being not symmetric, but skewed.

Depending on the behaviour of the Lévy measure $\nu$, or equivalently, on the behaviour of the Lévy exponent $\psi$, we obtain several types of limits for $Y_{T}$. All the limits are of course self-similar with stationary increments. We observe a phase transition depending on the parameters of the model, the limit process may be an $\alpha$-stable process with dependent increments ( $\alpha$ depends on $\nu$ ) or a stable Lévy process with index of stability which may be either $1+\gamma$ or smaller, depending on $\nu$.

For example, consider the case when $\Lambda$ is the standard independently scattered symmetric $\alpha$-stable random measure with Lebesgue control measure (i.e., $\nu(d x)=$ $\frac{\text { const }}{|x|^{1+\alpha}} d x$ and $\psi(x)=|x|^{\alpha}, 0<\alpha<2$ ).

- If $\alpha>1+\gamma$ then $F_{T}=T^{1-\gamma / \alpha}$ and for any $\tau>0$ the process $Y_{T}$ converges in law in $\mathcal{C}[0, \tau]$ to an $\alpha$-stable process with dependent increments, which is of the form constant times the process

$$
Y(t)=\int_{0}^{\infty} \int_{0}^{\infty}\left(r_{+} \wedge t-(r-u)_{+} \wedge t\right) u^{-\frac{2+\gamma}{\alpha}} M_{\alpha}(d r d u)
$$

where $M_{\alpha}$ is a symmetric $\alpha$-stable random measure on $\mathbb{R}_{+}^{2}$ with Lebesgue control measure. The integral is understood in the sense of [13]. The process $Y$ is selfsimilar with self-similarity index $H=1-\frac{\gamma}{\alpha}$, it has stationary increments and it is $\alpha$-stable, hence it may be thought of as yet another extension of fractional Brownian motion. 
- If $0<\alpha<1+\gamma$, then with the norming $F_{T}=T^{1 / \alpha}$ we have

$$
Y_{T} \stackrel{\text { f.d.d. }}{\Rightarrow} K \xi^{(\alpha)},
$$

where $\xi^{(\alpha)}$ is a symmetric $\alpha$-stable Lévy process and $K$ is some finite constant. ( $\stackrel{\text { f.d.d. }}{\Rightarrow}$ stands for convergence of finite dimensional distributions.)

- In the critical case $\alpha=1+\gamma$ we also have convergence (1.11) but the larger norming $F_{T}=T^{\frac{1}{\alpha}} \log T$. The appearance of the logarithm term is typical for the critical cases in many models.

Another simple example covered by our techniques is the following:

- Suppose that $\nu$ is a finite measure such that

$$
\int_{\mathbb{R}}|x|^{\kappa} \nu(d x)<\infty
$$

for some $\kappa>1+\gamma$. For example, $\Lambda$ can be a difference of two homogeneous Poisson random measures on $\mathbb{R}^{2}$. In this case the norming is $F_{T}=T^{\frac{1}{1+\gamma}}$ and the limit process is an $(1+\gamma)$-stable Lévy process. Note that the latter result corresponds to the one obtained in [9] in the discrete time setting.

In the next section we formulate our results in their general form. Depending on the interplay of the Lévy measure $\nu$ and the trawl function $g$, in the limit we obtain either the process $Y$ given by (1.10) or stable Lévy processes.

The paper is organised as follows: in Section 2 we recall some of the basic notions and we state the results. Section 3 contains the proofs. There we start with the general scheme, later applying it to prove our theorems.

Notation. By $C, C_{1}, C_{2}, \ldots$ we denote generic positive constants, whose value is not important to us. These constants may be different in different formulas. To help the reader we often write $C_{1}, C_{2}, \ldots$ to indicate that the constant changes from line to line.

$\stackrel{\text { f.d.d. }}{\Rightarrow}$ denotes convergence of finite dimensional distributions.

$C([0, \tau])$ with $\tau>0$ stands for the space of continuous functions from $[0, \tau]$ to $\mathbb{R}$.

\section{Results}

We assume that $\nu$ is a symmetric Lévy measure on $\mathbb{R}$. That is, $\nu$ is symmetric and satisfies (1.8). We consider a homogeneous Lévy basis $\Lambda$ on $\mathbb{R}^{2}$ corresponding to $\nu$, that is a family $(\Lambda(A))_{A \in \mathcal{E}}$ of real-valued random variables where $\mathcal{E}$ denotes the class of Borel subsets of $\mathbb{R}^{2}$ with finite Lebesgue measure. $\Lambda$ satisfies the following conditions:

1. $\Lambda$ is an independently scattered random measure, i.e., for any $A_{1}, A_{2}, \ldots \in \mathcal{E}$ with $A_{j} \cap A_{i}=\emptyset$ if $i \neq j, \Lambda\left(A_{1}\right), \Lambda\left(A_{2}\right), \ldots$ are independent and if additionally $\bigcup_{j=1}^{\infty} A_{j} \in \mathcal{E}$, then

$$
\Lambda\left(\bigcup_{j=1}^{\infty} A_{j}\right)=\sum_{j=1}^{\infty} \Lambda\left(A_{j}\right) \quad \text { a.s. }
$$

The series on the right converges almost surely.

2. For any $A \in \mathcal{E}$

$$
\mathbb{E} \exp (i \theta \Lambda(A))=\exp (-|A| \psi(\theta)), \quad \theta \in \mathbb{R},
$$

where $|A|$ denotes the Lebesgue measure of $A$ and $\psi$ is the Lévy exponent corresponding to $\nu$ :

$$
\psi(\theta)=\int_{\mathbb{R}}(1-\cos (\theta u)) \nu(d u) .
$$

$\psi$ has this simple form because we have assumed the symmetry of $\nu$. Also, in our setting there is no drift or diffusion part. 
Integrals of deterministic functions with respect to general Lévy bases were defined and studied in [12]. In our simple case, if a measurable function $f: \mathbb{R}^{2} \rightarrow \mathbb{R}$ satisfies

$$
\left.\int_{\mathbb{R}^{2}} \int_{\mathbb{R}}(u f(x))^{2} \wedge 1\right) \nu(d u) d x<\infty
$$

then the integral $I(f)=\int_{\mathbb{R}^{2}} f(x) \Lambda(d x)$ is well defined and

$$
\mathbb{E} \exp (i \theta I(f))=\exp \left(-\int_{\mathbb{R}^{2}} \int_{\mathbb{R}}(1-\cos (\theta u f(x))) \nu(d u) d x\right)
$$

(see [RR] and [11] Appendix B.1.5).

In particular, if $\Lambda$ is a symmetric $\alpha$-stable random measure, denoted by $M_{\alpha}$, that is corresponding to, $\psi(x)=|x|^{\alpha}$, and $\nu(d x)=\frac{C_{\alpha}}{|x|^{\alpha}}$, then $I(f)=\int_{\mathbb{R}^{2}} f d M_{\alpha}$ is the integral considered in [13]. In this case $I(f)$ is well defined if

$$
\int_{\mathbb{R}^{2}}|f(x)|^{\alpha} d x<\infty
$$

and

$$
\mathbb{E} \exp \left(i \theta \int_{\mathbb{R}^{2}} f d M_{\alpha}\right)=\exp \left(-\int_{\mathbb{R}^{2}}|f(x)|^{\alpha} d x\right) .
$$

We consider the trawl process described in the introduction. Suppose that $g:[0, \infty) \rightarrow$ $[0, \infty)$ is a continuous, integrable, strictly decreasing function. We define

$$
\begin{gathered}
A:=\{(x, y): x \leq 0, y \leq g(-x)\}, \\
A_{t}=\{(x, y): x \leq t, 0 \leq y \leq g(t-x)\}
\end{gathered}
$$

and set

$$
X_{t}=\Lambda\left(A_{t}\right), \quad t \geq 0
$$

For $T \geq 1$ we put

$$
Y_{T}(t)=\frac{1}{F_{T}} \int_{0}^{T t} X_{s} d s, \quad t \geq 0
$$

where $F_{T}$ is an appropriate norming, which will be specified later. Our basic assumption on the trawl function $g$ is the following.

Assumption (G). Assume that the trawl function $g$ is continuous, integrable, strictly decreasing, continuously differentiable on $(0, \infty)$ and its derivative satisfies

$$
\lim _{x \rightarrow \infty} x^{2+\gamma}\left|g^{\prime}(x)\right|=C_{g}
$$

for some $\gamma \in(0,1)$ and $C_{g}>0$.

Example 2.1. The function $g(x)=\frac{C}{(1+x)^{1+\gamma}}$ satisfies Assumption (G). For this function the proofs can be somewhat simplified since $g$ satsifies additionally

$$
\sup _{x>0} x^{2+\gamma}\left|g^{\prime}(x)\right| \leq C_{1} .
$$

Now we are ready to state our main results.

\subsection{Long range dependence regime}

Theorem 2.2. Suppose that assumption (G) is satisfied and that there exists $1+\gamma<\alpha<2$ and $C_{\psi}>0$ such that

$$
\lim _{|x| \rightarrow \infty} \frac{\psi(x)}{|x|^{\alpha}}=C_{\psi}
$$


Moreover, assume that there exists $\kappa>1+\gamma$ such that

$$
\int_{|y| \geq 1}|y|^{\kappa} \nu(d y)<\infty .
$$

Let $Y_{T}$ be given by (2.7) with

$$
F_{T}=T^{\frac{\alpha-\gamma}{\alpha}}
$$

Then, for any $\tau>0$, the processes $Y_{T}$ converge in law in $\mathcal{C}([0, \tau])$, as $T \rightarrow \infty$, to the process $K Y$, where $Y$ is defined by (1.10) and $K=\left(C_{\psi} C_{g}\right)^{1 / \alpha}$.

Remark 2.3. Whether or not condition (2.9) holds depends only on the behaviour of the Lévy measure $\nu$ near 0 since for any $\epsilon>0$ the function

$$
u \mapsto \int_{|x|>\epsilon}(1-\cos (u x)) \nu(d x)
$$

is bounded. If near zero $\nu$ has a density $h(x)$ such that

$$
\lim _{x \rightarrow 0}|x|^{1+\alpha} h(x)=C
$$

for some finite positive $C$ and $\alpha \in(0,2)$, then (2.9) is satisfied.

Remark 2.4. For $\alpha=2$ (i.e. when $\Lambda$ is a homogeneous Gaussian random measure) one can prove a result similar to the one of Theorem 2.2. In this case the limit process turns out to be fractional Brownian motion with Hurst coefficient $1-\gamma / 2$. Therefore, we may think of our limit process $Y$ as a yet another extension of fractional Brownian motion to the realm of stable processes.

Remark 2.5. We have written a basic code to simulate the process $Y$. Figure 1 shows pictures of sample paths obtained for various parameters $\alpha$ and $\gamma$. The interested reader may look up the Python code on the GitHub repository. ${ }^{1}$

The process $Y$ has dependent increments. Below we investigate the type of this dependence in terms of the dependence exponent introduced in [7], which is related to codifference of increments.

Recall that the dependence exponent of $Y$ is defined by

$$
\tilde{\kappa}=\inf _{z_{1}, z_{2} \in \mathbb{R}} \inf _{0 \leq w<v<p<t} \sup \left\{\gamma>0: D_{T}\left(z_{1}, z_{2} ; w, v, p, t\right)=o\left(T^{-\gamma}\right) \quad \text { as } \quad T \rightarrow \infty\right\}
$$

where

$$
\begin{aligned}
D_{T}\left(z_{1}, z_{2} ; w, v, p, t\right)=\mid & \log E e^{i\left(z_{1}(Y(v)-Y(w))+z_{2}(Y(T+t)-Y(T+p))\right.} \\
& -\log E e^{i z_{1}(Y(v)-Y(w))}-\log E e^{i z_{2}(Y(T+t)-Y(T+p))} \mid,
\end{aligned}
$$

see Definition 2.5 in [7]. Note that $D_{T}$ is the codifference of the corresponding increments. We have the following proposition.

Proposition 2.6. Assume that $1<1+\gamma<\alpha$ and let $Y$ be given by (1.10). Then for any $z_{1}, z_{2} \in \mathbb{R} \backslash\{0\}, 0 \leq w<v<p<t$ we have

$$
0<\lim _{t \rightarrow \infty} T^{\gamma}\left|D_{T}\left(z_{1}, z_{2} ; w, v, p, t\right)\right|<\infty
$$

hence the dependence exponent of $Y$ is equal to $\gamma$.

The proof of this proposition is given at the end of the paper, in Section 3.7.

\footnotetext{
${ }^{1}$ https://github.com/lukasz-treszczotko/trawl_processes_limits.
} 
Limit theorems for integrated trawl processes with symmetric Lévy bases
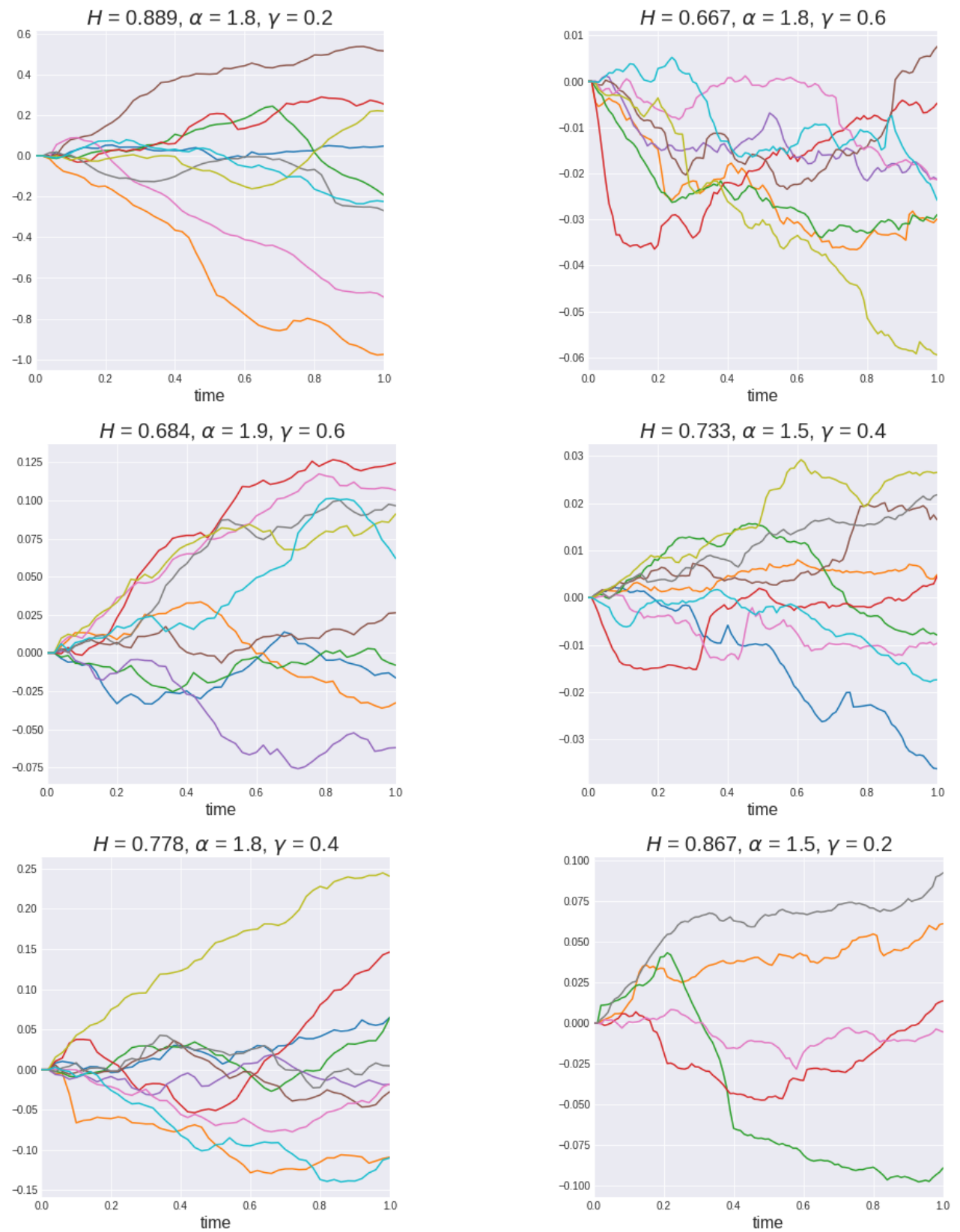

Figure 1: Simulated sample path trajectories of $Y$ for various pairs of $\alpha$ and $\gamma$

\subsection{Independent increments regime}

Theorem 2.7. Assume (G) and either

(i)

$$
\psi(u) \leq C|u|^{\kappa} \wedge|u|^{\alpha}, \quad u \in \mathbb{R}
$$

for some $2 \geq \kappa>1+\gamma, 0 \leq \alpha<1+\gamma$ and finite constant $C>0$, or

(ii) suppose that $\psi$ is nondecreasing on $[0, \infty)$,

$$
\int_{\mathbb{R}} \psi(u)|u|^{-2-\gamma} d u<\infty
$$


Limit theorems for integrated trawl processes with symmetric Lévy bases

and

$$
\sup _{u \geq 0} u^{2+\gamma}\left|g^{\prime}(u)\right| \leq C
$$

for some finite constant $C>0$.

Set

$$
F_{T}=T^{\frac{1}{1+\gamma}}
$$

Then for $Y_{T}$ given by (2.7) we have

$$
Y_{T} \stackrel{\text { f.d.d. }}{\Rightarrow} K \xi^{(1+\gamma)}, \quad \text { as } T \rightarrow \infty,
$$

where $\xi^{(1+\gamma)}$ denotes a symmetric $(1+\gamma)$-stable Lévy process and $K^{1+\gamma}=$ $C_{g} \int_{0}^{\infty} \psi(u) u^{-2-\gamma} d u$.

Remark 2.8. (a) Note that (2.16) implies (2.17). Condition (2.17) is slightly weaker, but in order to prove convergence under this assumption, we need to assume something more about the trawl function $g$.

(b) If

$$
\int_{\{|x|<1\}}|x|^{\alpha} \nu(d x)<\infty
$$

and (2.10) is satsified for $2 \geq \kappa>1+\gamma>\alpha \geq 0$, then using $|1-\cos (x)| \leq 2 \wedge x^{2} \leq 2|x|^{\delta}$ for any $0 \leq \delta \leq 2$ we obtain

$$
\begin{aligned}
\psi(u) & =\int_{\{|x|<1\}}(1-\cos (u x)) \nu(d x)+\int_{\{|x| \geq 1\}}(1-\cos (u x)) \nu(d x) \\
& \leq 2|u|^{\alpha} \int_{\{|x|<1\}}|x|^{\alpha} \nu(d x)+2 \nu(\{|x| \geq 1\}) \wedge\left(|u|^{\kappa} \int_{\{|x| \geq 1\}}|x|^{\kappa} \nu(d x)\right),
\end{aligned}
$$

hence (2.16) holds. In particular, if $\nu$ is a finite measure and satisfies (2.10), then (2.16) holds. Similarly as in Remark 2.3, if near zero $\nu$ has density satisfying (2.12) and (2.10) holds, then (2.16) is satisfied.

As a direct consequence of Theorem 2.7 we obtain the following result.

Example 2.9. If $\Lambda=N^{(1)}-N^{(2)}$, where $N^{(1)}$ and $N^{(2)}$ are two independent Poisson random measures on $\mathbb{R}^{2}$ with Lebesgue intensity measure, then the processes $Y_{T}$ converge in the sense of finite-dimensional distributions to a symmetric $(1+\gamma)$-stable Lévy process multiplied by a constant. In this case $\nu=\lambda\left(\delta_{1}+\delta_{-1}\right)$ for some $\lambda>0$ and $\psi(x)=2 \lambda(1-\cos (x))$. This result is a symmetrized continuous time analogue of the discrete time result of [9].

Theorem 2.10. Assume that (G) is satisfied and that there exist $0<\alpha<1+\gamma$ and a finite constant $C_{\alpha}>0$ such that

$$
\lim _{x \rightarrow 0} \frac{\psi(x)}{|x|^{\alpha}}=C_{\alpha}
$$

Furthermore, assume that there exist $C>0$ and $0 \leq \kappa<1+\gamma$ such that

$$
\psi(u) \leq C\left(1 \vee|u|^{\kappa}\right), \quad u \in \mathbb{R}
$$

Let $Y_{T}$ be defined by (2.7) with

$$
F_{T}=T^{\frac{1}{\alpha}}
$$

Then

$$
Y_{T} \stackrel{\text { f.d.d. }}{\Rightarrow} K \xi^{(\alpha)}, \quad \text { as } T \rightarrow \infty,
$$

where $K=\left(C_{\alpha} g(0)\right)^{1 / \alpha}$ and $\xi^{(\alpha)}$ is a symmetric $\alpha$-stable Lévy process. 
Let us now see how these general theorems work in the case of symmetric $\alpha$-stable random measures.

Example 2.11. Suppose that $\Lambda$ is a homogeneous and symmetric $\alpha$-stable random measure on $\mathbb{R}^{2}$ with $\alpha \in(0,2)$. We also assume that $(\mathbf{G})$ is staisfied. This case corresponds to

$$
\psi(x)=|x|^{\alpha}, \quad x \in \mathbb{R}
$$

and

$$
\nu(d x)=\frac{c_{\alpha}}{|x|^{\alpha+1}}, \quad x \in \mathbb{R} .
$$

- If $\alpha>1+\gamma$, then (2.10) holds for any $1+\gamma<\kappa<\alpha$, hence, the assumptions of Theorem 2.2 are satisfied, and with the norming $F_{T}=T^{1-\gamma / \alpha}$, for any $\tau>0$, the process $Y_{T}$ converges in law in $\mathcal{C}([0, \tau])$ to the process $K Y$, where $K$ is some finite constant and $Y$ is given by (1.10).

- If $\alpha<1+\gamma$, then the assumptions of Theorem 2.10 are satisfied and with the normalization $F_{T}=T^{1 / \alpha}$, the process $Y_{T}$ converges in the sense of finite-dimensional distributions to symmetric $\alpha$-stable Lévy process multiplied by a constant.

In the next theorem we will discuss the critical case $\alpha=1+\gamma$. As usual, the critical case is somewhat more complicated, thus we only consider a particular form of $g$.

Theorem 2.12. Assume that $\Lambda$ is a homogeneous symmetric $\alpha$-stable random measure on $\mathbb{R}^{2}$. Also, suppose that

$$
g(x)=\frac{1}{(1+x)^{1+\gamma}}, \quad x \geq 0,
$$

where $0<\gamma<1$. Let $Y_{T}$ be defined by (2.7) with

$$
F_{T}=T^{1 / \alpha} \log (T) .
$$

Then

$$
Y_{T} \stackrel{\text { f.d.d. }}{\Rightarrow} K \xi^{(\alpha)}, \quad \text { as } T \rightarrow \infty,
$$

where $K$ is a positive constant and $\xi^{(\alpha)}$ is a symmetric $\alpha$-stable Lévy process.

Thus, in the case of $\alpha$-stable random measures we have a phase transition: for large $\alpha(\alpha>1+\gamma)$ the limit process has dependent increments, while for small $\alpha(\alpha<1+\gamma)$ the limit process has independent increments. In the critical case $(\alpha=1+\gamma)$ the limit process also has independent increments but the norming differs by a logarithmic factor. This type of phase transition and existence of two regimes - one in which the limit process has independent increments and another one in which the increments are dependent, along with the logarithmic factor in the norming in the critical case is a typical behavior, also observed in other models. See for example [7] and [8] for a model with behaviour of this type, related to occupation time processes of branching particle systems.

\section{Proofs}

\subsection{General scheme}

In all the proofs we show convergence of finite-dimensional distributions by proving convergence of the corresponding characteristic functions. In Theorem 2.2 we additionally show tightness in $\mathcal{C}([0, \tau])$ for all $\tau>0$. We start with some general calculations used in all the cases.

First we write the process $Y_{T}$ in a different form, given by the lemma below. 
Lemma 3.1. Let $Y_{T}$ be given by (2.7). Then

$$
Y_{T}(t)=\frac{1}{F_{T}} \int_{\mathbb{R}^{2}}\left(\left(x+g^{-1}(y)\right)_{+} \wedge(T t)-x_{+} \wedge(T t)\right) \mathbb{1}_{\{0 \leq y \leq g(0)\}} \Lambda(d x, d y)
$$

Proof. It is immediate to see that

$$
\begin{aligned}
\int_{0}^{t} \mathbb{1}_{A_{s}}(x, y) d s & =\int_{0}^{t} \mathbb{1}_{\left\{x \leq s \leq g^{-1}(y)+x\right\}} d x \mathbb{1}_{\{0 \leq y \leq g(0)\}} \\
& =\left(\left(g^{-1}(y)+x\right)_{+} \wedge t-x_{+} \wedge t\right) \mathbb{1}_{\{0 \leq y \leq g(0)\}} .
\end{aligned}
$$

Hence (3.1) follows from the Fubini theorem for Lévy bases (see Theorem 3.1 in [2]). Note that this theorem can be applied directly in the case $\int_{\mathbb{R}}|y| \wedge|y|^{2} \nu(d y)<\infty$. If we do not assume $\int_{\{|y|>1\}}|y| \nu(d y)<\infty$, then we can decompose

$$
\Lambda=\Lambda_{1}+\Lambda_{2}
$$

where $\Lambda_{1}$ and $\Lambda_{2}$ are independent Lévy bases corresponding to Lévy measures $\nu_{1}$ and $\nu_{2}$, respectively, where

$$
\begin{aligned}
& \nu_{1}(B)=\nu(B \cap\{x:|x|<1\}), \\
& \nu_{2}(B)=\nu(B \cap\{x:|x| \geq 1\})
\end{aligned}
$$

for $B$ a Borel set in $\mathbb{R}$. Then $\Lambda_{1}$ satisfies the assumptions of Theorem 3.1 in [2] and $\Lambda_{2}$ can be written as

$$
\Lambda_{2}=\sum_{i} \eta_{i} \delta_{\left(x_{i}, y_{i}\right)},
$$

where $\left(x_{i}, y_{i}\right)$ are points of a Poisson random measure on $\mathbb{R}^{2}$ with Lebesgue intensity measure, multiplied by $\nu_{2}\left(\mathbb{R}^{2}\right)$ and $\eta_{i}$ are i.i.d. random variables with law $\nu_{2}(\cdot) / \nu_{2}\left(\mathbb{R}^{2}\right)$, independent of the Poisson random measure. The trawl function is non-decreasing and integrable, thus

$$
\sup _{0 \leq s \leq T t} \mathbb{1}_{A_{s}}(x, y) \leq \mathbb{1}_{A_{0} \cup[0, T t] \times[0, g(0)]}(x, y) .
$$

Only a finite number of points $\left(x_{i}, y_{i}\right)$ of the Poisson random measure belong to $A_{0} \cup$ $[0, g(0)] \times[0, T t]$, hence we can exchange the order of integration with respect to $d s$ and $\Lambda_{2}$ as well and (3.1) follows.

Note that in some of the proofs it will be convenient to use the decomposition (3.3) of $\Lambda$. Then

$$
Y_{T}=Y_{T, 1}+Y_{T, 2},
$$

where $Y_{T, 1}$ and $Y_{T, 2}$, are independent processes of the form (2.7), corresponding to $\Lambda_{1}$ and $\Lambda_{2}$, respectively. We also denote the corresponding characteristic exponents by

$$
\begin{aligned}
& \psi_{1}(\theta)=\int_{\{|x|<1\}}(1-\cos (\theta x)) \nu(d x), \quad \theta \in \mathbb{R} \\
& \psi_{2}(\theta)=\int_{\{|x| \geq 1\}}(1-\cos (\theta x)) \nu(d x), \quad \theta \in \mathbb{R} .
\end{aligned}
$$

As the next step we write the characteristic function of $Y_{T}$. We need some additional notation. Denote

$$
f(t, r, u):=r_{+} \wedge t-(r-u)_{+} \wedge t=\int_{0}^{t} \mathbb{1}_{[r-u, r]}(s) d s \quad t, r, u \geq 0 .
$$

We have the following lemma describing the characteristic function of finite-dimensional distributions of $Y_{T}$. 
Lemma 3.2. Fix $T>0, a_{1}, \ldots, a_{n} \in \mathbb{R}, 0 \leq t_{1} \leq \ldots \leq t_{k}<+\infty$ and denote

$$
h_{T}(r, u)=\sum_{j=1}^{n} a_{j} f\left(T t_{j}, r, u\right), \quad r, u \geq 0 .
$$

Then, for $Y_{T}$ defined by (2.7) we have

$$
\mathbb{E} \exp \left(i \sum_{j=1}^{k} a_{j} Y_{T}\left(t_{j}\right)\right)=\exp \left(-\int_{\mathbb{R}_{+}^{2}} \psi\left(\frac{1}{F_{T}} h_{T}(r, u)\right)\left|g^{\prime}(u)\right| d r d u\right)
$$

where $\psi$ is the Lévy exponent (2.2).

Proof. By Lemma 3.1, (2.4) and (2.2) we have

$$
\begin{aligned}
& \mathbb{E} \exp \left(i \sum_{j=1}^{k} a_{j} Y_{T}\left(t_{j}\right)\right) \\
& \quad=\exp \left\{-\int_{\mathbb{R}^{2}} \psi\left(\frac{1}{F_{T}} \sum_{j=1}^{n} a_{j}\left[\left(g^{-1}(y)+x\right) \wedge\left(T t_{j}\right)-x_{+} \wedge\left(T t_{j}\right)\right] \mathbb{1}_{0<y<g(0)}\right) d x d y\right\}
\end{aligned}
$$

Next we substitute $u=g^{-1}(y)$ and $r=x+g^{-1}(y)$. We also observe that if $r \leq 0$ we have $\left.\left(r_{+} \wedge\left(t_{j} T\right)-(r-u)_{+} \wedge\left(t_{j} T\right)\right)\right) \mathbb{1}_{\{u>0\}}=0$. Hence (3.11) follows.

The formula (3.11) will be our starting point of the proofs of convergence of finite dimensional distributions in Theorems 2.2, 2.7 and 2.10. Let us denote by $I(T)$ the term in the exponent on the right hand side of (3.11),

$$
I(T)=\int_{\mathbb{R}_{+}^{2}} \psi\left(\frac{1}{F_{T}} h_{T}(r, u)\right)\left|g^{\prime}(u)\right| d u .
$$

By (3.11), to prove convergence of finite dimensional disributions it suffices to show that

$$
\lim _{T \rightarrow \infty} e^{-I(T)}=\mathbb{E} \exp \left(i \sum_{j=1}^{n} a_{j} \tilde{Y}\left(t_{j}\right)\right)
$$

where $\tilde{Y}$ is the corresponding limit process.

This will amount to proving convergence of $I(T)$.

\subsection{Auxiliary estimates and identities}

We will frequently use the following simple facts concerning $f$ and $h_{T}$.

Lemma 3.3. Let $f$ be given by (3.9) and $h_{T}$ as in Lemma 3.1. Then

(i)

$$
\begin{aligned}
0 \leq f(t, r, u) & \leq t \wedge u \wedge r & & r, u, t \geq 0 \\
f(t, r, u) & =0 & & \text { for } t \geq 0 \text { and } r>t+u \\
\left|h_{T}(r, u)\right| & \leq\left(\sum_{j=1}^{n}\left|a_{j}\right|\right) f\left(T t_{n}, r, u\right), & & r, u \geq 0 .
\end{aligned}
$$

(ii) If, additionally, we assume that $\kappa>1+\gamma>1$ then there exists a constant $C>0$ depending only on $\kappa$ and $\gamma$, such that for all $t \geq 0$ we have

$$
\int_{0}^{\infty} \int_{0}^{\infty}|f(t, r, u)|^{\kappa} u^{-2-\gamma} d u d r=C t^{\kappa-\gamma}
$$


Proof. Part (i) is a direct consequence of (3.9) and (3.10).

To prove (ii) observe that by (3.13) and (3.14) for $t=1$ we have

$$
\begin{aligned}
\int_{0}^{\infty} \int_{0}^{\infty}|f(1, r, u)|^{\kappa} u^{-2-\gamma} d r d u= & \int_{0}^{\infty} \int_{0}^{1+u}|f(1, r, u)|^{\kappa} u^{-2-\gamma} d r d u \\
\leq & \int_{0}^{1} \int_{0}^{1+u} u^{\kappa} u^{-2-\gamma} d r d u \\
& +\int_{1}^{\infty} \int_{0}^{1+u} u^{-2-\gamma} d r d u<+\infty
\end{aligned}
$$

since $\kappa>1+\gamma$. Now, using

$$
f(t, r, u)=t f(1, r / t, u / t)
$$

(3.16) follows by a simple substitution.

\subsection{Proof of Theorem 2.2}

First observe that by part (ii) of Lemma 3.3, (2.5) and (2.6) it follows that the process $Y$ given by (1.10) is well defined.

We will show convergence of finite-dimensional distributions and then establish tightness on any interval $[0, \tau], \tau>0$, which suffices to obtain the desired convergence (see Thm. 8.1 in [6]).

\section{Step 1. Convergence of finite dimensional distributions}

Fix any $a_{1}, \ldots, a_{n} \in \mathbb{R}$ and $0 \leq t_{1} \leq \ldots \leq t_{n}$ and recall the notation (3.12) and (3.10). Let us also denote

$$
h(r, u)=\sum_{j=1}^{n} a_{j} f\left(t_{j}, r, u\right)=\sum_{j=1}^{n} a_{j}\left(r_{+} \wedge t_{j}-(r-u)_{+} \wedge t_{j}\right), \quad r, u \geq 0 .
$$

Using (3.11), (3.12) and (2.6), to prove convergence of finite-dimensional distributions, we only have to show that

$$
\lim _{T \rightarrow \infty} I(T)=K^{\alpha} \int_{\mathbb{R}_{+}^{2}}|h(r, x)|^{\alpha} u^{-2-\gamma} d r d u
$$

for some finite positive constant $K$.

By (3.12), (3.10), (3.17) and recalling the definition of $F_{T}(2.11)$ we have

$$
\begin{aligned}
I(T) & =\int_{\mathbb{R}_{+}^{2}} T^{2} \psi\left(\frac{T}{F_{T}} h(r, u)\right)\left|g^{\prime}(T u)\right| d r d u \\
& =\int_{\mathbb{R}_{+}^{2}}\left(\frac{T}{F_{T}}\right)^{-\alpha} \psi\left(\frac{T}{F_{T}} h(r, u)\right) T^{2+\gamma}|g(T u)| d r d t .
\end{aligned}
$$

By (2.8) and (2.9) we see that the integrand converges pointwise to the integrand on the right hand side of (3.18). Therefore, to prove (3.18) it remains to justify the passage to the limit under the integral.

We will use the decomposition (3.6), which corresponds to $\psi=\psi_{1}+\psi_{2}$, where $\psi_{1}$ and $\psi_{2}$ are given by (3.7) and (3.8), respectively. We write

$$
I(T)=I_{1}(T)+I_{2}(T)
$$

where $I_{1}(T)$ and $I_{2}(T)$ are defined by (3.12) with $\psi$ replaced by $\psi_{1}$ and $\psi_{2}$, respectively. 
We will show that

$$
\begin{aligned}
& \lim _{T \rightarrow \infty} I_{1}(T)=K^{\alpha} \int_{\mathbb{R}_{+}^{2}}|h(r, x)|^{\alpha} u^{-2-\gamma} d r d u, \\
& \lim _{T \rightarrow \infty} I_{2}(T)=0 .
\end{aligned}
$$

This will imply

$$
Y_{T, 1} \stackrel{\text { f.d.d. }}{\Rightarrow} K Y \quad \text { and } \quad Y_{T, 2} \stackrel{\text { f.d.d. }}{\Rightarrow} 0 .
$$

As the limit of $Y_{T, 2}$ is deterministic, $Y_{T, 2}(t)$ converges to 0 in probability for any $t>0$, hence (3.24) implies the desired convergence of finite-dimensional distributions of $Y_{T}$.

Observe, that by the estimate $1-\cos (\theta x) \leq(\theta x)^{2}$, (3.7) and (2.9) we have

$$
0 \leq \psi_{1}(x) \leq C\left(|x|^{\alpha} \wedge|x|^{2}\right) \leq C|x|^{\alpha} .
$$

We may assume that $\kappa$ in the assumptions of the Theorem satisfies $1+\gamma<\kappa<\alpha$, since if (2.10) holds for some $\kappa$, then it also holds for smaller $\kappa$. In particular, $\kappa<2$. Then, using $(1-\cos (x \theta)) \leq 2|\theta x|^{\kappa}$ (3.8) and (1.8) we have

$$
\psi_{2}(x) \leq C\left(|x|^{\kappa} \wedge 1\right) \leq C|x|^{\kappa} .
$$

Since $\psi_{2}$ is bounded and $\alpha>1+\gamma>0$ we have

$$
\begin{aligned}
& \lim _{|x| \rightarrow \infty} \frac{\psi_{1}(x)}{|x|^{\alpha}}=\lim _{|x| \rightarrow \infty} \frac{\psi(x)}{|x|^{\alpha}}=C_{\psi}, \\
& \lim _{|x| \rightarrow \infty} \frac{\psi_{2}(x)}{|x|^{\alpha}}=0 .
\end{aligned}
$$

Moreover, by Assumption $(\mathbf{G})$ there exists $D>0$ such that

$$
\sup _{u \geq D}\left|g^{\prime}(u)\right| u^{2+\gamma} \leq 2 C_{g},
$$

and we may therefore write

$$
I_{i}(T)=A_{i}(T)+B_{i}(T), \quad i=1,2,
$$

where

$$
\begin{aligned}
& A_{i}(T)=\int_{0}^{D / T} \int_{0}^{\infty}\left(\frac{T}{F_{T}}\right)^{-\alpha} \psi_{i}\left(\frac{T}{F_{T}} h(r, u)\right) T^{2+\gamma}\left|g^{\prime}(T u)\right| d r d u \quad i=1,2 \\
& B_{i}(T)=\int_{0}^{\infty} \int_{0}^{\infty} \mathbb{1}_{\left(\frac{D}{T}, \infty\right)}(u)\left(\frac{T}{F_{T}}\right)^{-\alpha} \psi_{i}\left(\frac{T}{F_{T}} h(r, u)\right) T^{2+\gamma}\left|g^{\prime}(T u)\right| d r d u \quad i=1,2 .
\end{aligned}
$$

Let us consider $A_{1}(T)$ first. By (3.25) we have

$$
A_{1}(T) \leq C \int_{0}^{\frac{D}{T}} \int_{0}^{\infty}|h(r, u)|^{\alpha} T^{2+\gamma}\left|g^{\prime}(T u)\right| d r d u .
$$

Then for $T>1$ by (3.17), (3.9) and Lemma 3.3 (i) we obtain

$$
\begin{aligned}
A_{1}(T) & \leq C_{1} \int_{0}^{\frac{D}{T}} \int_{0}^{t_{n}+\frac{D}{T}} u^{\alpha} T^{2+\gamma}\left|g^{\prime}(T u)\right| d u \\
& =C_{1}\left(t_{n}+D\right) T^{1+\gamma-\alpha} \int_{0}^{D} u^{\alpha}\left|g^{\prime}(u)\right| d u \\
& \leq C_{1}\left(t_{n}+D\right) D^{\alpha} g(0) T^{1+\gamma-\alpha} \rightarrow 0 .
\end{aligned}
$$


Similarly, using $\kappa<\alpha,\left(\frac{T}{F_{T}}\right)^{-\alpha} \leq\left(\frac{T}{F_{T}}\right)^{-\kappa}$ for $T \geq 1$ and (3.26) we have

$$
A_{2}(T) \leq C \int_{0}^{\frac{D}{T}} \int_{0}^{\infty}|h(r, u)|^{\kappa} T^{2+\gamma}\left|g^{\prime}(T u)\right| d r d u
$$

and the same argument as above shows that $A_{2}(T) \rightarrow 0$.

Now let us proceed to $B_{1}(T)$. By (3.27) and Assumption (G) the integrand in (3.32) with $i=1$ converges to $C_{\psi} C_{g}|h(r, x)|^{\alpha} u^{-2-\gamma}$. Moreover, by (3.25) and (3.29), it is bounded by

$$
C_{2}|h(r, u)|^{\alpha} u^{-2-\gamma} .
$$

By (3.17), (3.9), part (ii) of Lemma 3.3 and the fact that $\alpha>1+\gamma$ the latter function is integrable on $\mathbb{R}_{+}^{2}$, hence we can pass to the limit under the integral sign, and (3.22) follows.

It remains to consider $B_{2}(T)$. Using (3.26) and (3.29), $1+\gamma<\kappa<\alpha$, and again Lemma 3.3 (ii) for $T \geq 1$ we have

$$
B_{2}(T) \leq C\left(\frac{T}{F_{T}}\right)^{\kappa-\alpha} \int_{\mathbb{R}_{+}^{2}}|h(r, u)|^{\kappa} u^{-2-\gamma} d r d u \leq C\left(\frac{T}{F_{T}}\right)^{\kappa-\alpha} \rightarrow 0 .
$$

This finishes the proof of (3.23). We have proved (3.24).

Step 2. Tightness.

Now we continue to establish tightness in $\mathcal{C}([0, \tau])$ for any $\tau>0$.

Let us consider the sequence $\left(Y_{T, 2}\right)$ first. We are going to use Theorem 12.3 in [6]. Without loss of generality we may assume that $\alpha>\kappa>1+\gamma$ and $T \geq 1$. Since for each $T \geq 1$ the process $Y_{T, 2}$ has stationary increments, one only has to show that there exist $C>0, \beta \geq 0, \epsilon>0$ such that

$$
\mathbb{P}\left(\left|Y_{T, 2}(t)\right| \geq \lambda\right) \leq \frac{C}{\lambda^{\beta}} t^{1+\epsilon}, \quad T \geq 1, t \geq 0, \lambda>0 .
$$

We will use the following estimate, valid for any real valued random variable $\xi$

$$
\mathbb{P}(|\xi|>\lambda) \leq \lambda \int_{-2 / \lambda}^{2 / \lambda}(1-\mathbb{E} \exp (i \theta \xi)) d \theta, \quad \lambda>0
$$

By (3.11), recalling (3.9) we have

$$
\mathbb{E} \exp \left(i \theta Y_{T, 2}(t)\right)=\exp \left(-\int_{0}^{\infty} \int_{0}^{\infty} T^{2} \psi_{2}\left(\frac{\theta T}{F_{T}} f(t, r, u)\right)\left|g^{\prime}(T u)\right| d r d u\right) .
$$

Hence, using (3.26), (2.11), the simple inequality $1-e^{-x} \leq x$ and the fact that for $T \geq 1$ we have $\left(T / F_{T}\right)^{\kappa} \leq\left(T / F_{T}\right)^{\alpha}=T^{\gamma}$ it follows that

$$
\begin{aligned}
1-\mathbb{E} \exp \left(i \theta Y_{T, 2}(t)\right) & \leq C \int_{0}^{\infty} \int_{0}^{\infty} T^{2}\left|\frac{\theta T}{F_{T}} f(t, r, u)\right|^{\kappa}\left|g^{\prime}(T u)\right| d r d u \\
& =C|\theta|^{\kappa} \int_{0}^{\infty} \int_{0}^{\infty}|f(t, r, u)|^{\kappa} T^{2+\gamma}\left|g^{\prime}(T u)\right| d r d u \\
& =C|\theta|^{\kappa}\left(J_{1}(T)+J_{2}(T)\right),
\end{aligned}
$$

where

and

$$
J_{1}(T)=\int_{0}^{1} \int_{0}^{\infty}|f(t, r, u)|^{\kappa} T^{2+\gamma}\left|g^{\prime}(T u)\right| d r d u
$$

$$
J_{2}(T)=\int_{1}^{\infty} \int_{0}^{\infty}|f(t, r, u)|^{\kappa} T^{2+\gamma}\left|g^{\prime}(T u)\right| d r d u
$$


Notice that for $u \in(1, \infty)$ and all $T$ sufficiently large $T^{2+\gamma}\left|g^{\prime}(T u)\right| \leq C|u|^{-2-\gamma}$ for some finite positive constant $C$. Thus, by Lemma 3.3 (ii) we have

$$
J_{2}(T) \leq C_{1} t^{\kappa-\gamma}
$$

for all $T$ large and some finite constant $C_{5}$. Now, let $\epsilon>0$ be such that $\kappa>1+\gamma+\epsilon$. By (3.9) and (3.13), and then using $\int_{0}^{\infty} \mathbb{1}_{[r-u, r]}(s) d r=u$ for $s, r>0$, we see that

$$
\begin{aligned}
J_{1}(T) & \leq \int_{0}^{1} \int_{0}^{\infty}\left(\int_{0}^{t} \mathbb{1}_{[r-u, r]}(s) d s\right) t^{\epsilon} u^{\kappa-1-\epsilon} T^{2+\gamma}\left|g^{\prime}(T u)\right| d r d u \\
& =t^{\epsilon} \int_{0}^{1} t u u^{\kappa-1-\epsilon} T^{2+\gamma}\left|g^{\prime}(T u)\right| d u \\
& =t^{1+\epsilon} T^{1+\gamma+\epsilon-\kappa} \int_{0}^{T} u^{\kappa-\epsilon}\left|g^{\prime}(u)\right| d u \\
& \leq t^{1+\epsilon} \int_{0}^{\infty} u^{\kappa-\epsilon}\left|g^{\prime}(u)\right| d u
\end{aligned}
$$

Let $D$ be as in (3.29), then

$$
J_{1}(T) \leq t^{1+\epsilon}\left(D^{\kappa-\epsilon} \int_{0}^{D}\left|g^{\prime}(u)\right| d u+2 C_{g} \int_{D}^{\infty} u^{\kappa-2-\gamma-\epsilon} d u\right) \leq C t^{1+\epsilon},
$$

since the first integral is bounded by $g(0)$, and the second is finite thanks to the choice of $\epsilon$. Combining (3.38), (3.37), (3.36) with (3.34) yields (3.33) (here $\beta=\kappa$ ) for all $t \geq 0$ and all $T$ large enough. This finishes the proof of tightness of $Y_{T, 2}$ in $\mathcal{C}([0, \tau])$

The proof of tightness of $Y_{T, 1}$ is similar. We have an analogue of (3.36) with $\alpha$ instead of $\kappa$ and the same argument works. In this case $\epsilon=\alpha-1-\gamma$.

Combined with convergence of finite dimensional distributions this implies convergence of $Y_{T}$ in $C([0, \tau])$ for any $\tau>0$.

\subsection{Proof of Theorem 2.7}

We will show convergence of finite-dimensional distributions by proving the convergence their characteristic functions.

According to the general scheme, we fix any $a_{1}, \ldots, a_{n} \in \mathbb{R}, 0 \leq t_{1} \ldots \leq t_{n}$ and we start with formula (3.11). To prove the theorem it suffices to show that for $I(T)$ defined by (3.12) and (3.10) we have

$$
\lim _{T \rightarrow \infty} I(T)=K^{1+\gamma} \int_{0}^{\infty}|a(r)|^{1+\gamma} d r
$$

where

$$
a(r)=\sum_{j=1}^{n} a_{j} \mathbb{1}_{\left[0, t_{j}\right]}(r) .
$$

Recalling the definition of $h_{T}$ (see (3.10)) and substituting $r^{\prime}=\frac{r}{T}, u^{\prime}=\frac{u}{F_{T}}$ and then $s^{\prime}=\frac{(s-r)}{u} \frac{T}{F_{T}}$ we obtain

$$
\begin{aligned}
I(T) & =\int_{0}^{\infty} \int_{0}^{\infty} T F_{T} \psi\left(\frac{T}{F_{T}} \int_{0}^{\infty} a(s) \mathbb{1}_{\left[r-\frac{u F_{T}}{T}, r\right]}(s) d s\right)\left|g^{\prime}\left(F_{T} u\right)\right| d r d u \\
& =\int_{0}^{\infty} \int_{0}^{\infty} \psi\left(u \int_{-1}^{0} a\left(r+\frac{u}{T} F_{T} s\right) d s\right) F_{T}^{2+\gamma}\left|g^{\prime}\left(F_{T} u\right)\right| d r d u
\end{aligned}
$$

where in the last equality we also used $T=F_{T}^{1+\gamma}$. 
By Assumption (G) it is now clear that the integrand in (3.41) converges pointwise to $C_{g} \psi(u a(r)) u^{-2-\gamma}$. Also notice, that making the substitution $u^{\prime}=u a(r)$ we have

$$
\int_{0}^{\infty} \int_{0}^{\infty} C_{g} \psi(u a(r)) u^{-2-\gamma} d u d r=C_{g} \int_{0}^{\infty} \psi(u) u^{-2-\gamma} d u \int_{0}^{\infty}|a(r)|^{1+\gamma} d r .
$$

The integral with respect to $u$ on the right hand side of (3.42) is finite by (2.16) or (2.17), hence (3.39) will follow provided we can justify passing to the limit under the integrals.

Now the proof forks into two parts depending on whether we assume (i) or (ii) in the formulation of Theorem 2.7.

Consider first the case when (i) is satisfied. Using Assumption (G) choose $D>0$ such that (3.29) holds. Suppose that $T$ is such that $T>1$ and $T>D$. Observing that since the support of $a$ is $\left[0, t_{n}\right]$ and hence the integrand in (3.41) is equal to zero if $r>t_{n}+u \frac{F_{T}}{T}$ we write

$$
I(T)=I_{1}(T)+I_{2}(T)+I_{3}(T),
$$

where

$$
\begin{aligned}
& I_{1}(T)=\int_{0}^{\frac{D}{F_{T}}} \int_{0}^{t_{n}+u \frac{F_{T}}{T}} \psi\left(u \int_{-1}^{0} a\left(r+\frac{u}{T} F_{T} s\right) d s\right) F_{T}^{2+\gamma}\left|g^{\prime}\left(F_{T} u\right)\right| d r d u, \\
& I_{2}(T)=\int_{\frac{D}{F_{T}}}^{\frac{T}{F_{T}}} \int_{0}^{t_{n}+u \frac{F_{T}}{T}} \psi\left(u \int_{-1}^{0} a\left(r+\frac{u}{T} F_{T} s\right) d s\right) F_{T}^{2+\gamma}\left|g^{\prime}\left(F_{T} u\right)\right| d r d u, \\
& I_{3}(T)=\int_{\frac{T}{F_{T}}}^{\infty} \int_{0}^{t_{n}+u \frac{F_{T}}{T}} \psi\left(u \int_{-1}^{0} a\left(r+\frac{u}{T} F_{T} s\right) d s\right) F_{T}^{2+\gamma}\left|g^{\prime}\left(F_{T} u\right)\right| d r d u .
\end{aligned}
$$

We will show that $I_{2}$ has a non-trivial limit and $I_{1}$ and $I_{3}$ converge to 0 . By (2.16) we have

$$
\begin{aligned}
I_{1}(T) & \leq C \int_{0}^{D / F_{T}} \int_{0}^{t_{n}+\frac{u F_{T}}{T}}\left|u\|a\|_{\infty}\right|^{\kappa} F_{T}^{2+\gamma}\left|g^{\prime}\left(F_{T} u\right)\right| d r d u \\
& \leq C_{1}\left(t_{n}+D\right) \int_{0}^{D / F_{T}} u^{\kappa} F_{T}^{2+\gamma}\left|g^{\prime}\left(F_{T} u\right)\right| d r d u \\
& =C_{1}\left(t_{n}+D\right) F_{T}^{1+\gamma-\kappa} \int_{0}^{D} u^{\kappa}\left|g^{\prime}(u)\right| d u \\
\leq & C_{1}\left(t_{n}+D\right) D^{\kappa} g(0) F_{T}^{1+\gamma-\kappa} \rightarrow 0,
\end{aligned}
$$

since we have assumed that $\kappa>1+\gamma$.

Now we consider $I_{2}(T)$. The integrand converges pointwise to $C_{g} \psi(u a(r)) u^{-2-\gamma}$. Moreover, by assumption (2.16) and the fact that the support of $a$ is $\left[0, t_{n}\right]$, for $D / F_{T} \leq$ $u \leq T / F_{T}$ we have

$$
\psi\left(u \int_{-1}^{0} a\left(r+\frac{u}{T} F_{T} s\right) d s\right) F_{T}^{2+\gamma}\left|g^{\prime}\left(F_{T} u\right)\right| \leq C \mathbb{1}_{\left[0, t_{n}+1\right]}(r)\left(u^{\kappa} \wedge u^{\alpha}\right) u^{-2-\gamma} .
$$

The latter function is integrable on $\mathbb{R}_{+}^{2}$. Hence, using also (3.42) we see that

$$
\lim _{T \rightarrow \infty} I_{2}(T)=K^{1+\gamma} \int_{0}^{\infty}|a(r)|^{1+\gamma} d r
$$

Now we proceed to $I_{3}(T)$. Observe that since $|a(s)| \leq\|a\|_{\infty} \mathbb{1}_{\left[0, t_{n}\right]}(s)$ we have

$$
\left|u \int_{-1}^{0} a\left(r+\frac{u}{T} F_{T} s\right) d s\right| \leq \int_{\mathbb{R}}|a(s)| d s \frac{T}{F_{T}} \leq\|a\|_{\infty} t_{n} \frac{T}{F_{T}} .
$$


Thus, using (2.16) we can estimate

$$
\begin{aligned}
I_{3}(T) & \leq C \int_{T / F_{T}}^{\infty}\left(t_{n}+u \frac{F_{T}}{T}\right)\left(\frac{T}{F_{T}}\right)^{\alpha} u^{-2-\gamma} d u \\
& =\left(\frac{T}{F_{T}}\right)^{\alpha-1-\gamma} \int_{1}^{\infty}\left(t_{n}+u\right) u^{-2-\gamma} d u \rightarrow 0
\end{aligned}
$$

by asumption $\alpha<1+\gamma$ and the form of $F_{T}$.

From (3.43), (3.44), (3.46) and (3.48) we obtain (3.39) in case (i) which completes the proof of convergence of finite dimensional distributions in this case.

Now consider the case (ii) in the formulation of Theorem 2.7 is satisfied. We again have (3.41) and (3.43). Now for $I_{1}+I_{2}$ we can proceed in a similar way as for $I_{2}$ in case (i). The only difference is that instead of (3.45) for $0 \leq u \leq \frac{T}{F_{T}}$, we use

$$
\psi\left(u \int_{-1}^{0} a\left(r+\frac{u}{T} F_{T} s\right) d s\right) F_{T}^{2+\gamma}\left|g^{\prime}\left(F_{T} u\right)\right| \leq C \mathbb{1}_{\left[0, t_{n}+1\right]}(r) \psi\left(\|a\|_{\infty} u\right) u^{-2-\gamma},
$$

since we now assume that $\psi$ is nondecreasing on $\mathbb{R}_{+}$. Similarly as above we obtain that $I_{1}(T)+I_{2}(T)$ converge, as $T \rightarrow \infty$, to the right hand side of (3.39)

For $I_{3}$ we again use (3.47) and monotonicity of $\psi$ on $\mathbb{R}_{+}$obtaining

$$
\begin{aligned}
I_{3}(T) & \leq C \int_{T / F_{T}}^{\infty}\left(t_{n}+u \frac{F_{T}}{T}\right) \psi\left(\frac{T}{F_{T}}\|a\|_{\infty} t_{n}\right) u^{-2-\gamma} d u \\
& =\left(\frac{T}{F_{T}}\right)^{-1-\gamma} \psi\left(\frac{T}{F_{T}}\|a\|_{\infty} t_{n}\right) \int_{1}^{\infty}\left(t_{n}+u\right) u^{-2-\gamma} d u .
\end{aligned}
$$

It now suffices to notice that $T^{-1-\gamma} \psi(T)$ converges to 0 as $T \rightarrow \infty$, since by the fact that $\psi$ is nondecreasing

$$
\frac{1}{1+\gamma} \psi(T) T^{-1-\gamma}=\int_{T}^{\infty} \psi(T) x^{-2-\gamma} d x \leq \int_{T}^{\infty} \psi(x) x^{-2-\gamma} d x .
$$

The last integral converges to 0 by (2.17). This proves that $I_{3}(T)$ converges to 0 . The proof in case (ii) is complete.

\subsection{Proof of Theorem 2.10}

We use the decomposition (3.6). Using the estimate $1-\cos (\theta x) \leq(\theta x)^{2}$, (3.7) and (1.8) we have $\psi_{1}(x) \leq C x^{2}$. This together with the assumption (2.22) implies

$$
\psi_{1}(x) \leq C|x|^{2} \wedge|x|^{\kappa}
$$

The assumptions of Theorem 2.7, in which we take $\bar{\alpha}=\kappa$ and $\bar{\kappa}=2$, are satisfied for $\psi_{1}$ and the process $\left(F_{T} / T^{1+\gamma}\right) Y_{T, 1}$ converges in the sense of finite dimensional distributions. $F_{T}=T^{\frac{1}{\alpha}}$ with $\alpha<1+\gamma$ hence the above implies that

$$
Y_{T, 1} \stackrel{\text { f.d.d. }}{\Rightarrow} 0 \text {. }
$$

And therefore also $Y_{T, 1}(t)$ converges to 0 in probability for any $t \geq 0$.

From now on we may therefore assume that $\nu(\{|x| \leq 1\})=0$ and $\psi=\psi_{2}$. In what follows we omit the index 2. Observe that in this case $\psi$ is bounded since $\nu$ is finite (cf. (1.8)) and from assumption (2.21) it follows that

$$
\psi(x) \leq C\left(|x|^{\alpha} \wedge 1\right)
$$


Take any $a_{1}, \ldots, a_{n} \in \mathbb{R}$ and $0 \leq t_{1} \leq \ldots \leq t_{n}$ in $\mathbb{R}_{+}$. According to the general scheme (cf. (3.11)) we need to show that for $I(T)$ given by (3.12) and $a$ by (3.40) we have

$$
\lim _{T \rightarrow \infty} I(T)=K^{\alpha} \int_{0}^{\infty}|a(r)|^{\alpha} d r .
$$

Using (3.12), (3.10), (3.9), (3.14) and substituting $r^{\prime}=\frac{r-u}{T}$ we rewrite $I(T)$ as

$$
I(T)=I_{1}(T)+I_{2}(T),
$$

where

$$
\begin{aligned}
& I_{1}(T)=\int_{0}^{\infty} \int_{-u / T}^{0} T \psi\left(\frac{1}{F_{T}} \sum_{j=1}^{n} a_{j} f\left(T t_{j}, T r+u, u\right)\right)\left|g^{\prime}(u)\right| d r d u \\
& I_{2}(T)=\int_{0}^{\infty} \int_{0}^{t_{n}} T \psi\left(\frac{1}{F_{T}} \sum_{j=1}^{n} a_{j} f\left(T t_{j}, T r+u, u\right)\right)\left|g^{\prime}(u)\right| d r d u .
\end{aligned}
$$

Observe that, by (3.9), for $u \geq 0$ and $r \geq 0$ we have

$$
\lim _{T \rightarrow \infty} f\left(T t_{j}, T r+u, u\right)=\lim _{T \rightarrow \infty} \int_{0}^{T t_{j}} \mathbb{1}_{[T r, T r+u]}(s) d s=u \mathbb{1}_{\left[0, t_{j}\right)}(r),
$$

and

$$
f\left(T t_{j}, T r+u, u\right) \leq u .
$$

Using (3.52), (3.49) and (3.55) we have

$$
I_{1}(T) \leq C \int_{0}^{\infty} u\left(\left(\frac{u \sum_{j=1}^{n}\left|a_{j}\right|}{F_{T}}\right)^{\alpha} \wedge 1\right)\left|g^{\prime}(u)\right| d u \longrightarrow 0,
$$

since the function under the integral converges pointwise to 0 and is bounded by $u\left|g^{\prime}(u)\right|$, which is integrable by Assumption (G).

Now we proceed to $I_{2}(T)$. By (3.53), (3.54), (2.21) and (2.23) we see that

$$
\lim _{T \rightarrow \infty} T \psi\left(\frac{1}{F_{T}} \sum_{j=1}^{n} a_{j} f\left(T t_{j}, T r+u, u\right)\right)\left|g^{\prime}(u)\right|=C_{\alpha}|a(r)|^{\alpha}\left|g^{\prime}(u)\right| \quad \text { a.e. }
$$

and by (3.49)

$$
T \psi\left(\frac{1}{F_{T}} \sum_{j=1}^{n} a_{j} f\left(T t_{j}, T r+u, u\right)\right)\left|g^{\prime}(u)\right| \leq C u^{\alpha}\left|g^{\prime}(u)\right| .
$$

The function on the right hand side is integrable on $\mathbb{R}_{+} \times\left[0, t_{n}\right]$. Hence

$$
\lim _{T \rightarrow \infty} I_{2}(T)=\int_{0}^{\infty} \int_{0}^{t_{n}}|a(r)|^{\alpha}\left|g^{\prime}(u)\right| d r d u=C_{\alpha} g(0) \int_{0}^{\infty}|a(r)|^{\alpha} d r .
$$

From (3.51), (3.56) and (3.57) we obtain (3.50), thus finishing the proof of the theorem.

\subsection{Proof of Theorem 2.12}

Take any $a_{1}, \ldots, a_{n} \in \mathbb{R}$ and $0 \leq t_{1} \leq \ldots \leq t_{n} \geq 0$. Recall the general formula for the characteristic function of finite dimensional distributions of $Y_{T}$ (3.11) and the 
notation (3.10), (3.9), (3.12) and (3.40). By Lemma 3.2, to prove the desired convergence of finite dimensional distributions it suffices to show

$$
\lim _{T \rightarrow \infty} I(T)=K^{\alpha} \int_{0}^{\infty}|a(r)|^{\alpha} d r .
$$

Since $\psi(x)=|x|^{\alpha}$, using (3.10), (3.9) and then substituting $r^{\prime}=\frac{r}{T}$ and $s^{\prime}=\frac{s}{T}$ we may rewrite $I_{T}$ as

$$
\begin{aligned}
I(T) & =\int_{0}^{\infty} \int_{0}^{\infty} F_{T}^{-\alpha}\left|\sum_{j=1}^{n} a_{j} \int_{0}^{\infty} \mathbb{1}_{[r-u, r]}(s) \mathbb{1}_{\left[0, T t_{j}\right]}(s) d s\right|^{\alpha}\left|g^{\prime}(u)\right| d r d u \\
& =\int_{0}^{\infty} \int_{0}^{\infty} T F_{T}^{-\alpha}\left|T \int_{0}^{\infty} a(s) \mathbb{1}_{\left[r-\frac{u}{T}, r\right]}(s) d s\right|^{\alpha}\left|g^{\prime}(u)\right| d r d u
\end{aligned}
$$

Now we use the form of $F_{T}$ and of $g$, then make a change of variables $s^{\prime}=\frac{(r-s)}{u} T$, and then, finally, substitute $u^{\prime}=u / \log T$, obtaining

$$
\begin{aligned}
I(T) & =\frac{(1+\gamma)}{\log T} \int_{0}^{\infty} \int_{0}^{\infty} u^{\alpha}\left|\int_{0}^{1} a(r-s u / T) d s\right|^{\alpha}(1+u)^{-2-\gamma} d r d u \\
& =(1+\gamma) \int_{0}^{\infty} \int_{0}^{\infty} \frac{(u \log T)^{\alpha}}{(1+u \log T)^{1+\alpha}}\left|\int_{0}^{1} a(r-s u \log T / T) d s\right|^{\alpha} d r d u .
\end{aligned}
$$

Now we write

$$
I(T)=(1+\gamma)\left(I_{1}(T)+I_{2}(T)+I_{3}(T)\right),
$$

where

$$
\begin{aligned}
& I_{1}(T)=\int_{0}^{1} \int_{0}^{\infty} \ldots d r d u \\
& I_{2}(T)=\int_{1}^{T / \log T} \int_{0}^{\infty} \ldots d r d u \\
& I_{3}(T)=\int_{T / \log T}^{\infty} \int_{0}^{\infty} \ldots d r d u
\end{aligned}
$$

where ... stands for the function under the integral in (3.59). Let us consider first $I_{2}(T)$. We make a change of variables $u^{\prime}=\frac{\log u}{\log T}$ obtaining

$$
\begin{aligned}
& I_{2}(T) \\
& \quad=\int_{0}^{1-\log \log T / \log T} \int_{0}^{\infty}\left(\frac{T^{u} \log T}{1+T^{u} \log T}\right)^{1+\alpha}\left|\int_{0}^{1} a\left(r-s T^{u} \log T / T\right) d s\right|^{\alpha} d r d u .
\end{aligned}
$$

Notice that $\log \log T / \log T$ goes to zero as $T \rightarrow \infty$. Moreover, we have pointwise convergence to $|a(r)|^{\alpha}$. We have $|a(r)| \leq C \mathbb{1}_{\left[0, t_{n}\right]}(r)$ for some finite constant $C$ hence the upper limit in the integral with respect to $r$ can be replaced by $t_{n}+1$, since for $r>t_{n}+1$ the function under the integral with respect to $d r d u$ vanishes. We may use the dominated convergence theorem obtaining

$$
\lim _{T \rightarrow \infty} I_{2}(T)=\int_{0}^{\infty}|a(r)|^{\alpha} d r
$$


Let us consider $I_{1}(T)$ next. We have

$$
\begin{aligned}
I_{1}(T) & =\int_{0}^{1} \int_{0}^{t_{n}+1} \frac{(u \log T)^{\alpha}}{(1+u \log T)^{1+\alpha}}\left|\int_{0}^{1} a(r-s u \log T / T) d s\right|^{\alpha} d r d u \\
& \leq\|a\|_{\infty}\left(1+t_{n}\right) \int_{0}^{1} \frac{(u \log T)^{\alpha}}{(1+u \log T)^{1+\alpha}} d u \\
& =C \int_{0}^{\log T} \frac{u^{\alpha}}{(1+u)^{\alpha+1}} \frac{1}{\log T} d u \\
& \leq C_{1}\left(\frac{1}{\log T} \int_{0}^{1} \frac{u^{\alpha}}{(1+u)^{\alpha+1}}+\frac{1}{\log T} \int_{1}^{\log T} \frac{1}{u} d u\right) \\
& \leq C_{2}\left(\frac{1}{\log T}+\frac{\log \log T}{\log T}\right) \rightarrow 0 .
\end{aligned}
$$

It remains to show that $I_{3}(T)$ also converges to 0 as $T \rightarrow \infty$. Taking into account that the support of $a$ is $\left[0, t_{n}\right]$, after a change of variables we have

$$
\begin{aligned}
I_{3}(T) & =\frac{1}{\log T} \int_{T}^{\infty}\left(\int_{0}^{t_{n}+u / T} \frac{u^{\alpha}}{(1+u)^{1+\alpha}}\left|\int_{0}^{\infty} a(r-s u / T) \mathbb{1}_{[0,1]}(s) d s\right|^{\alpha} d r\right) d u \\
& \leq \frac{1}{\log T} \int_{T}^{\infty}\left(\int_{0}^{\left(t_{n}+1\right) u / T} \frac{T^{\alpha}}{(1+u)^{1+\alpha}}\left|\frac{u}{T} \int_{0}^{\infty} \mathbb{1}_{\left[0, t_{n}\right]}(r-s u / T) d s\right|^{\alpha} d r\right) d u \\
& \leq C \frac{\left(1+t_{n}\right)}{\log T} \int_{T}^{\infty} \frac{u}{T} \frac{T^{\alpha}}{u^{\alpha+1}} d u \\
& =\frac{C_{1}}{\log T} \int_{T}^{\infty} \frac{T^{\alpha-1}}{u^{\alpha}} d u \\
& =C_{2} \frac{1}{\log T} \rightarrow 0 .
\end{aligned}
$$

Combining (3.60)-(3.64) shows that (3.58) is satisfied. This finishes the proof of the theorem.

\subsection{Proof of Proposition 2.6}

Let $0 \leq w<v<p<t, T \geq 0$ and $z_{1}, z_{2} \in \mathbb{R}$. We will investigate the asymptotic behaviour of (2.14) as $T \rightarrow \infty$. To shorten the notation we will drop the arguments in the parenthesis and write it as $D_{T}$. Notice that (1.10) can be written as

$$
Y_{t}=\int_{0}^{\infty} \int_{0}^{\infty} \int_{0}^{\infty} \mathbf{1}_{[0, t]}(s) \mathbf{1}_{[r-u, r]}(s) d s u^{-\frac{2+\gamma}{\alpha}} M_{\alpha}(d r, d u) .
$$

Since $Y$ has stationary increments we can assume that $w=0$. We can also assume that $T \geq t+p+v$. Then

$$
\begin{aligned}
D_{T}= & \int_{0}^{\infty} \int_{0}^{\infty} \mid z_{1} \int_{0}^{\infty} \mathbf{1}_{[0, v]}(s) \mathbf{1}_{[r-u, r]}(s) d s \\
& +\left.z_{2} \int_{0}^{\infty} \mathbf{1}_{[p+T, t+T]}(s) \mathbf{1}_{[r-u, r]}(s) d s\right|^{\alpha} u^{-2-\gamma} d r d u \\
& -\int_{0}^{\infty} \int_{0}^{\infty}\left|z_{1} \int_{0}^{\infty} \mathbf{1}_{[0, v]}(s) \mathbf{1}_{[r-u, r]}(s) d s\right|^{\alpha} u^{-2-\gamma} d r d u \\
& -\int_{0}^{\infty} \int_{0}^{\infty}\left|z_{2} \int_{0}^{\infty} \mathbf{1}_{[p+T, t+T]}(s) \mathbf{1}_{[r-u, r]}(s) d s\right|^{\alpha} u^{-2-\gamma} d r d u
\end{aligned}
$$


Limit theorems for integrated trawl processes with symmetric Lévy bases

Note that

$$
\int_{0}^{\infty} \mathbf{1}_{[0, v]}(s) \mathbf{1}_{[r-u, r]}(s) d s \neq 0 \quad \text { if and only if } \quad r<u+v
$$

and

$$
\int_{0}^{\infty} \mathbf{1}_{[p+T, t+T]}(s) \mathbf{1}_{[r-u, r]}(s) d s \neq 0 \quad \text { if and only if } \quad p+T<r<u+t+T .
$$

Always $u+v<u+T+t$, hence

$$
\begin{aligned}
D_{T}= & \int_{p+T-v}^{\infty} d u \int_{p+T}^{u+v} d r\left(\mid z_{1} \int_{0}^{\infty} \mathbf{1}_{[0, v]}(s) \mathbf{1}_{[r-u, r]}(s) d s\right. \\
& +\left.z_{2} \mathbf{1}_{[p+T, t+T]}(s) \mathbf{1}_{[r-u, r]}(s) d s\right|^{\alpha} \\
& -\left|z_{1} \int_{0}^{\infty} \mathbf{1}_{[0, v]}(s) \mathbf{1}_{[r-u, r]}(s) d s\right|^{\alpha} \\
& \left.-\left|z_{2} \int_{0}^{\infty} \mathbf{1}_{[p+T, t+T]}(s) \mathbf{1}_{[r-u, r]}(s) d s\right|^{\alpha}\right) u^{-2-\gamma}
\end{aligned}
$$

(if at least one of the integrals with respect to $d s$ vanishes, then the difference above also vanishes). Substitute $u^{\prime}:=u-T$ and $r^{\prime}:=r-T$ to get

$$
\begin{aligned}
D_{T}= & \int_{p-v}^{\infty} d u \int_{p}^{u+v} d r\left(\mid z_{1} \int_{0}^{\infty} \mathbf{1}_{[0, v]}(s) \mathbf{1}_{[r-u, r+T]}(s) d s\right. \\
& +\left.z_{2} \mathbf{1}_{[p+T, t+T]}(s) \mathbf{1}_{[r-u, r+T]}(s) d s\right|^{\alpha} \\
& -\left|z_{1} \int_{0}^{\infty} \mathbf{1}_{[0, v]}(s) \mathbf{1}_{[r-u, r+T]}(s) d s\right|^{\alpha} \\
& \left.-\left|z_{2} \int_{0}^{\infty} \mathbf{1}_{[p+T, t+T]}(s) \mathbf{1}_{[r-u, r+T]}(s) d s\right|^{\alpha}(u+T)^{-2-\gamma}\right) .
\end{aligned}
$$

On the set of integration we have $r-u<v$ and thus $r-u<p+T$. Therefore

$$
\begin{aligned}
D_{T}= & \int_{p-v}^{\infty} d u \int_{p}^{u+v} d r\left(\left|z_{1} \int_{0}^{\infty} \mathbf{1}_{[r-u, v]}(s) d s+z_{2}((r \wedge t)-p)\right|^{\alpha}\right. \\
& \left.-\left|z_{1} \int_{0}^{\infty} \mathbf{1}_{[r-u, v]}(s) d s\right|^{\alpha}-\left|z_{2}((r \wedge t)-p)\right|^{\alpha}\right)(u+T)^{-2-\gamma} .
\end{aligned}
$$

We split the above integral as follows:

$$
D_{T}=\underbrace{\int_{p-v}^{t} d u \int_{p}^{u+v} d r \ldots}_{J_{1}(T)}+\underbrace{\int_{t}^{\infty} d u \int_{p}^{t} d r \ldots}_{J_{2}(T)}+\underbrace{\int_{t}^{\infty} d u \int_{t}^{u} d r \ldots}_{J_{3}(T)}+\underbrace{\int_{t}^{\infty} d u \int_{u}^{u+v} d r \ldots \ldots}_{J_{4}(T)}
$$

Clearly

$$
\left|J_{1}(T)\right| \leq C_{1} T^{-2-\gamma}
$$

for some constant $C_{1}$ independent of $T$. Next,

$$
\begin{aligned}
J_{2}(T) & =\int_{t}^{\infty} d u \underbrace{\int_{p}^{t} d r\left(\left|z_{1} v+z_{2}(r-p)\right|^{\alpha}-\left|z_{1} v\right|^{\alpha}-\left|z_{2}(r-p)\right|^{\alpha}\right)}_{C_{2}\left(\alpha, p, t, v, z_{1}, z_{2}\right)}(u+T)^{-2-\gamma} \\
& =C_{2}\left(\alpha, p, t, v, z_{1}, z_{2}\right) T^{1-2-\gamma} \int_{t / T}^{\infty}(u+1)^{-2-\gamma} d u,
\end{aligned}
$$


which means that

$$
\lim _{T \rightarrow \infty} T^{1+\gamma} J_{2}(T) \rightarrow C_{2}\left(\alpha, p, t, v, z_{1}, z_{2}\right) \int_{0}^{\infty}(u+1)^{-2-\gamma} d u
$$

For $J_{3}$ we have

$$
\begin{aligned}
J_{3}(T) & =\int_{t}^{\infty} d u \int_{t}^{u} d r \underbrace{\left(\left|z_{1} v+z_{2}(t-p)\right|^{\alpha}-\left|z_{1} v\right|^{\alpha}-\left|z_{2}(t-p)\right|^{\alpha}\right)}_{C_{3}\left(\alpha, p, t, v, z_{1}, z_{2}\right)}(u+T)^{-2-\gamma} \\
& =C_{3}\left(\alpha, p, t, v, z_{1}, z_{2}\right) T^{-\gamma} \int_{t / T}^{\infty}(u-t / T)(u+1)^{-2-\gamma} d u .
\end{aligned}
$$

Hence

$$
\lim _{T \rightarrow \infty} T^{\gamma} J_{3}(T)=C_{3}\left(\alpha, p, t, v, z_{1}, z_{2}\right) \int_{0}^{\infty} u(u+1)^{-2-\gamma} d u .
$$

Note that $C_{3}\left(\alpha, p, t, v, z_{1}, z_{2}\right) \neq 0$ if $z_{1} \neq 0$ and $z_{2} \neq 0$. Finally

$$
\begin{aligned}
J_{4}(T) & =\int_{t}^{\infty} d u \int_{u}^{u+v} d r\left(\left|z_{1}(v-r+u)+z_{2}(t-p)\right|^{\alpha}-\left|z_{1}(v-r+u)\right|^{\alpha}\right. \\
\left.-\left|z_{2}(t-p)\right|^{\alpha}\right)(u+T)^{-2-\gamma} & \underbrace{\infty}_{C_{4}\left(\alpha, p, t, v, z_{1}, z_{2}\right)} d u \underbrace{\int_{0}^{v} d r\left(\left|z_{1} r+z_{2}(t-p)\right|^{\alpha}-\left|z_{1} r\right|^{\alpha}-\left|z_{2}(t-p)\right|^{\alpha}\right)}_{0}(u+T)^{-2-\gamma} \\
& =\int_{t}^{\infty}
\end{aligned}
$$

and similarly as in $J_{3}(T)$ we have

$$
\lim _{T \rightarrow \infty} T^{1+\gamma} J_{4}(T)=C_{4}\left(\alpha, p, t, v, z_{1}, z_{2}\right) \int_{0}^{\infty}(u+1)^{-2-\gamma} d u .
$$

From (3.70)-(3.74) we obtain (2.15), which finishes the proof of Proposition 2.6.

\section{References}

[1] O. E. Barndorff-Nielsen. Stationary infinitely divisible processes. Brazilian Journal of Probability and Statistics, 25(3):294-322, 2011. MR-2832888

[2] O. E. Barndorff-Nielsen and A. Basse-O'Connor. Quasi Ornstein-Uhlenbeck processes. Bernoulli, 17(3):916-941, 2011. MR-2817611

[3] O. E. Barndorff-Nielsen, F. E. Benth, and A. E. D. Veraart. Recent advances in ambit stochastics with a view towards tempo-spatial stochastic volatility/intermittency. In Advances in mathematics of finance, volume 104 of Banach Center Publ., pages 25-60. Polish Acad. Sci. Inst. Math., Warsaw, 2015. MR-3363978

[4] O. E. Barndorff-Nielsen, F. E. Benth, and A. E. D. Veraart. Ambit stochastics, volume 88 of Probability Theory and Stochastic Modelling. Springer, Cham, 2018. MR-3839270

[5] O. E. Barndorff-Nielsen, A. Lunde, N. Shephard, and A. E. D. Veraart. Integer-valued trawl processes: A class of stationary infinitely divisible processes. Scandinavian Journal of Statistics, 41(3), 2014. MR-3249424

[6] P. Billingsley. Convergence of Probability Measures. John Wiley \& Sons, first edition, 1968. MR-0233396

[7] T. Bojdecki, L. Gorostiza, and A. Talarczyk. A long range dependence stable process and an infinite variance branching system. Ann. Probab., 35(2):500-527, 2007. MR-2308586

[8] T. Bojdecki, L. G. Gorostiza, and A. Talarczyk. Occupation time fluctuations of an infinitevariance branching system in large dimensions. Bernoulli, 13(1):20-39, 2007. MR-2307392 
Limit theorems for integrated trawl processes with symmetric Lévy bases

[9] P. Doukhan, A. Jakubowski, S. R. C. Lopes, and D. Surgailis. Discrete-time trawl processes. Stochastic Processes and their Applications, 129(4):1326-1348, 2019. MR-3926557

[10] D. Grahovac, M. S. Taqqu, and N. N. Leonenko. Intermittency of trawl processes. Statist. Probab. Lett., 137:235-242, 2018. MR-3776222

[11] V. Pipiras and M. S. Taqqu. Long-Range Dependence and Self-Similarity. Cambridge University Press, 2017. MR-3729426

[12] B. S. Rajput and J. Rosinski. Spectral representations of infinitely divisible processes. Probability Theory and Related Fields, 82(3):451-487, 1989. MR-1001524

[13] G. Samorodnitsky and S. Taqqu. Stable Non-Gaussian Random Processes. Stochastic Modelling. CRC Press, first edition, 1994. MR-1280932

[14] A. E. Veraart and E. D. Almut. Modeling, simulation and inference for multivariate time series of counts using trawl processes. J. Multivariate Anal., 169:110-129, 2019. MR-3875590

[15] R. L. Wolpert and M. S. Taqqu. Fractional Ornstein - Uhlenbeck Lévy processes and the Telecom process: Upstairs and downstairs. Signal Processing, 85(8):1523-1545, 2005. 